\title{
Elderly's Barriers and Requirements for Interactive TV
}

\author{
Mai Baunstrup and Lars Bo Larsen \\ Department of Electronic Systems, Aalborg University, Denmark \\ $\{\mathrm{mba}, 1 \mathrm{bl}\}$ @es. aau. dk
}

\begin{abstract}
This paper presents a study to identify the problems and experiences that the elderly have using interactive TV (iTV) services. The study comprised an in-depth qualitative interview series backed up with a questionnaire survey; a list with the elderly's interaction problems and the reasons for wanting or not wanting to use iTV services was developed. These findings in turn lead to the formulation of a set of user requirements. The paper presents the studies carried out and the resulting design recommendations for iTV services for the elderly. The recommendations also take cognitive and as physiological impairments into consideration. The recommendations propose on increased ease-of-use, transparency, colour schemes, familiarity and a reduced set of iTV services for the elderly. Ultimately, designers should aim at customizable interface profiles for future iTV devices and services to better accommodate the diversity of users and especially the elderly.
\end{abstract}

Keywords: Categories and Subject Descriptors H.5.2 Information Interfaces and Presentation (e.g., HCI) - User Interfaces, Evaluation/Methodology. General Terms: Human Factors, Measurement TV, iTV, Qualitative Study, Explorative Interview, Survey, Elderly.

\section{Introduction}

Television is changing its form from being a one-way communication channel to being a part of a networked media ecology [Fleury, 2012]. Television consumption is rapidly going from a monologue to becoming a dialogue in the growing development of interactive TV (iTV). The infrastructure is developing faster than the corresponding interfaces to access the services are. The expanded range of available iTV services induces more complex interaction paradigms and typically more devices such as set-top boxes, second screen and media streaming devices and their corresponding remote controls, etc.

This means that the challenge now is to provide an enhanced user experience [Cesar \& Chorianopoulos, 2008], [Tsekleves, et al., 2011]. To meet this challenge, it is necessary to better understand exactly who experience what problems when interacting with iTV services. A survey was carried out within this study aimed to reveal problems regarding the usage of the TV and iTV services. It revealed that $41 \%$ of participants younger than 60 years had difficulties in general compared to $56 \%$ of the participants above 60 . This survey will be discussed in more details later in this 
article. This finding supports the conclusions by [Madden, 2007] and [Schellenbach, et al., 2010]. For the senior citizens the interaction problems, navigating and using the new technologies, etc. are more pronounced.

For most populations in the world "population aging" are a fact, the elderly segment is growing very fast [Weil, 2006]. In the UK, senior citizens of 65 years and above watch $40 \%$ more TV than the national average and they watch the most television of any age group [Gauntlett \& Hill, 1999]. They also point out that the elderly segment is probably the most ignored when developing iTV content and the user interfaces for interaction. Different studies state that more research is missing in this area and there is a lack of studies on users' domestic activities of the home environment within the human-computer interaction field [Tsekleves, et al., 2011] [Obrist, et al., 2008].

It is therefore of interest to investigate, which barriers and problems especially senior citizens have interacting with their iTV setup and services and to identify the elderly's reasons for wanting or not wanting to use the emerging iTV services. This can in turn lead to the formulation of design guidelines and recommendations.

\subsection{Related Work}

A study reported in [Tsekleves, et al., 2011] into attitudes towards different types of media and the role of TV within the home environment applied a methodology where 27 families from the UK were interviewed four times in their homes over a duration of 3 years. The participants' age ranged from 8 to above 50 . The study revealed that TV consumption is closely associated with familiarity and ease of use for all age groups, especially for older adults. They also report that participants preferred the interactive features that helped them make decision about what to watch and found that it should require minimal effort. An experimental device (a second screen) was created to facilitate further exploration of attitudes, expectations and barriers surrounding iTV and digital media use in the home. They found that $96 \%$ of the participants liked the idea of being able to access a range of media (TV, photos, video) from the device and projecting this content to the TV.

In an ethnographic study from Austria [Obrist, et al., 2008] Obrist and colleagues categorized the participants into four groups depending on age and household structure. For the group denoted "Individuals with different social life circumstances, older than 55 years" Obrist concluded that; (a) attitudes and expectations changes with age over time; (b) and inflexibility increases; (c) ones interpretation of a given stimulus becomes more fixed; (d) ones goals of life and future plans, do affect models of thinking and attitudes towards the medium; (e) that elderly will most likely adopt iTV and for them, the burden to access the Internet through the TV is lower than buying and learning to interact with a computer. The study point out: "It will be essential to provide the elderly with easy-to-use interactive services, which allow them on one hand an extended usage of their TV and on the other hand a familiar TV-viewing experience". 


\subsection{The Current Study and Paper Outline}

A qualitative approach for investigating these issues is conducted to obtain an indepth understanding of the mental, social and physical factors in play in the daily use of iTV services for the elderly. The study will encompass explorative interviews with a number of senior citizens and apply a phenomenological analysis. Explorative interviews are a powerful method for conducting user studies as it allows deep investigation of the research question with test subjects. The interviews are supplemented with a broader survey to compare and validate the finding to other age groups.

The next section presents related work on the issues mentioned in the introduction; section 3 outlines some of the age-related issues leading to physiological and cognitive impairments; section 4 presents the qualitative study and the methodologies used, section 5 contains the resulting design guidelines/recommendations of TV and iTV functionalities and section 6 concludes the study.

\section{Physiological and Cognitive Effects of Ageing}

This section describes the physical and cognitive effects during late adulthood and derives requirements for iTV services for the elderly.

\subsection{Age Effects on the Visual and Haptic Senses}

The most important sense in this context is the visual: It takes longer time for older adults to process visual stimuli and for identifying an object. This process is enhanced; the weaker the stimulus is that the elderly is looking at [Stuart-Hamilton, 2001]. The field of vision also is getting smaller with age because elderly individuals cannot move their eyeball as far up as younger people can. A bigger problem is that a decline in the peripheral vision (width of the field of vision) occur from middle-aged and get much weaker from the age of 75 years of age [Stuart-Hamilton, 2001]. By the age of 60 the lens in the eye is completely incapable of accommodating to focus on objects at close distance [Whitbourne, 2002]. Opacities in the lens called cataracts are likely to occur in later adulthood, and interfere with vision by diffusing light as it passes through the lens, reducing the clarity of the image that reaches the retina [Whitbourne, 2002]. Also, a change in colour perception occurs causing the world to seem more yellow than it is. The sensitivity is greatest for the colours red, orange and yellow and it becomes harder to distinguish among green, blue and violet colours. This decline in colour perception generally occurs when reaching the eighties [Stuart-Hamilton, 2001].

The ability to detect details at different distances is denoted the visual acuity. After the fifties a progressive decline is happening until the age of 85 . At the age of 85 an $80 \%$ loss compared to the level achieved in the forties, that is a loss of visual acuity of about $2 \%$ per year [Whitbourne, 2002]. Similarly, in addition to a general decline in the auditory senses, the auditory acuity deteriorates and it becomes harder to pick out e.g. a human voice from background noise or a particular voice among many talkers. 
The somatosensory (touch) sense also declines with age [Goldstein, 2007]. Elderly has a higher touch-threshold, which means that a stronger stimulus of the skin is needed in order to be detected.

Cognitive Effects of Aging. Many changes happen in the way individuals experience their environments, when getting older. The brain weight decreases about 10-15\% during the normal ageing, which have serious potential consequence to psychological processes [Stuart-Hamilton, 2001]. Research shows that older adults may suffer brain deficits in one area but may make up for these deficits by increasing the activation of other brain regions [Whitbourne, 2002]. Both sensation and perception appear to be affected in significant ways by the aging process as discussed above. The change in sensation reduces the quality and quantity of information, so the individuals do not get as much information to base their bottom-up processing upon as younger people do [Whitbourne, 2002].

David Madden [Madden, 2007] states that most visual search involve a combination of bottom-up and top-down processing. The processing does not occur in entirely separate processing stages but instead interact to achieve performance. A suggestion made by Madden is that some parts of the top-down processing (attentional guidance) are preserved and older adults have an increased reliance of their top-down processing. Consequently, older adults have to rely on different types of routines, compensating for the losses in sensory and perceptual abilities, which will dominate the course of their everyday life [Whitbourne, 2002]. [Schellenbach, et al., 2010] states that spatial navigation skills are deteriorating over age, and this is due to a decline in sensorimotor functioning, spatial processing and the motivation to explore new environments.

\subsection{Physiological and Cognitive Requirements}

From the analysis of the ageing effects, the following observations should be taken into consideration when creating design guidelines for the elderly:

- Perceive the colours red, orange and yellow better than green blue and violet

- A decline in visual clarity

- A decline in the auditory acuity

- The touch-threshold is higher

- It takes longer time to perceive visual stimuli

- Blurred vision reduces clarity

- Incapability of focusing on objects at close range

- Uses primarily top-down processing

- Informational stimuli is reduced both in quality and quantity

- Age-related functions decline independently

These observations will be included into the proposed design recommendations. 


\section{Explorative Interviews}

The interviews were completed with twelve participants between 60 and 69 years, who were interviewed alone and in their own homes. The interviews took between 40 and 110 minutes and were conducted by one interviewer. The interviews were recorded on a Dictaphone. Seven females and five males participated. Five participants live in the countryside, five participants in Copenhagen and the last two live in the medium-sized city Aalborg. In Figure 4.2 shows a participant during the explorative interview.

The interview script consisted of 86 questions divided into five parts. Before creating the questions two small focus group interviews were conducted. The focus group interviews took place in a care home together with an activity centre, six persons above eighty and one above 60 participated. The group interviews acted as an exploration to get an idea of the topic, before formulating the questions in the interview script.

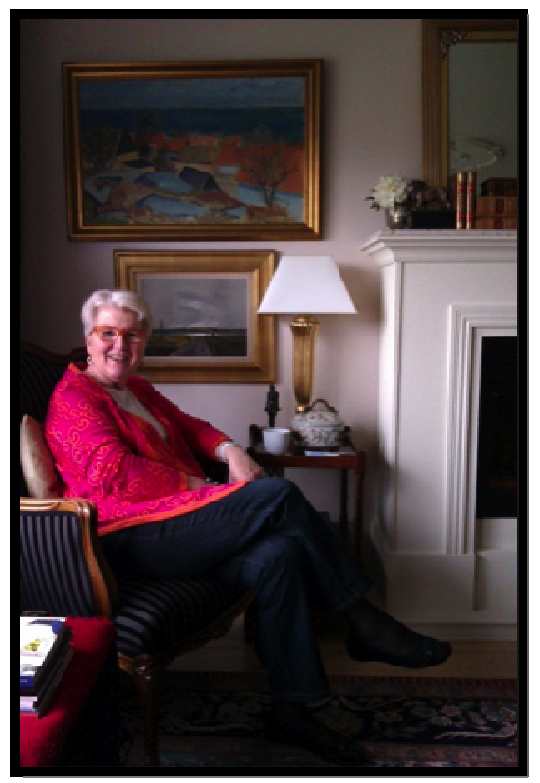

Fig. 1. A Participant in the explorative Interview

\subsection{Analysis of the Qualitative Data Collected from the Interviews}

The chosen method for analysing the qualitative data is the Five-phase cycle method described in [Yin, 2011]. These are: Step 1: Compiling, which includes transcription using meaning condensation [Kvale \& Brinkmann, 2009]. Steps 2 and 3: Disassembling and Reassembling (and Arraying). The disassembling procedure proceeds to break 
down the compiled data into smaller fragments or pieces, then creating labels or codes to the fragments or pieces. In the reassembling phase essential themes or codes are created for clustering and reorganize the disassembled data into different groupings. The final steps are interpreting and concluding, which are discussed below.

Interpretation and Conclusions. All comments about problems or issues regarding iTV services were collated. Each of the comments was given a label created to describe the kind of problem. This procedure was iterated three times.

First the question: "What kind of problems do people between 60 and 69 years of age experience with their home TV media set-up and what are their expectations about the use of iTV functions?" were addressed. Twelve labels were created and a further grouping lead to four categories:

1. Interaction problems: Set-up; Need for external support; Too many remote controls; Not as easy as before

2. Way of thinking: Too expensive; Distrust; I keep to the familiar; Confusing to me; I do not want to disturb anyone else

3. Cultural mismatch: Unclear terms from the TV service provider; Lack of information

4. Hardware problems: Annoyance induced by things I cannot do anything about

The label Set-up was the most frequent problem in the first category. Most of the participants had huge difficulties sorting the channel order either when setting up their new TV or when the cable signal supplier changed the TV signal. Quoting participant P2: "It is unnecessarily difficult to arrange channels into a logical sorted order". An example of Cultural mismatch: is given by $\mathrm{P} 1:$ "When we were told EPG existed, it was easy to use".

Next the question: "Which reasons do people between 60 and 69 years have for wanting or not wanting to use iTV services" is addressed. Twelve labels were created and further grouped into two categories:

1. Reasons for Wanting to Use iTV Services: Once I am aware of the existence of a service, I want to try it; I want to use the service and I know why; I want to use the service on certain conditions; I use the service now and I want to use it in a new way; I want to use the service but I have further usability requirements.

2. Reasons for Not Wanting to Use iTV services: I keep using the service in the familiar way until I'm forced to change; I do not want to use the service due to usability issues; I do not want to use the service and I do not need it; I do not want to use the service because it does not fit my behaviours/I have no time for it; I do not want to use the service due to my way of thinking; I do not want to use the service because it is interrupting or like spam.

Clearly, the reasons given for not wanting to use iTV services are related to strong preferences to the familiar and well known. Usability problems for some services are also a barrier. Another strong reason is that the interviewees simply do not see a need 
for a given service. For example, discussing the functions about commenting and recommending TV shows to others P6 noted: "No need for that function I already use $S M S$ " and P4 on the use of the electronic program guide (EPG): "Not now, but when we do not have a subscription on newspapers anymore, then we will use EPG".

The reason: "I do not want to use the service due to my way of thinking" was the second most common. For example P7 mentioned: "It is empty information, you cannot use the info for anything" about a Facebook-style "Like" function.

Validation of the Qualitative Research. Validity of qualitative research begins as the project is conceptualized and developed and continues throughout the research process. The five verification strategies described in [Morse, et al., 2002] are adopted in the current study. These are: Methodological coherence; appropriateness of the sample; concurrent collection and analysis of data; and theoretic thinking and theory development. Together all these five verification strategies contribute to and build reliability and validity thus ensuring rigor.

\subsection{Questionnaire Survey}

Following up on the interviews, a questionnaire was created to further explore the issues, compare the findings to other age groups and act as an additional validation of the interviews. The survey consisted of 24 questions derived from the interviews. The survey was handed out in a Bingo centre and 51 persons answered the questionnaires. Eleven of those were between 60 and 69.

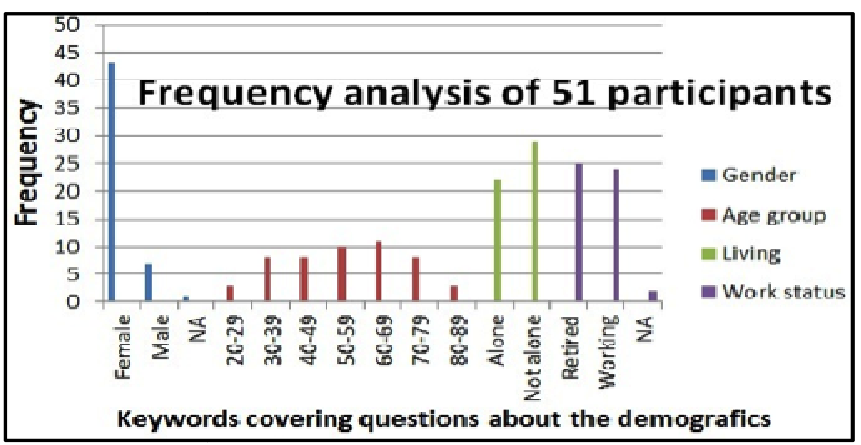

Fig. 2. Demographic distribution of questionnaire respondents

Figure 2 shows the demographic distribution of the respondents. Figure 3 below compares the survey with the interview results.

Eight key questions are shown in Figure 3; one question reveals whether or not they have difficulties with iTV or/and the TV set-up, six questions about specific iTV services and the last question is about using an Ipad as remote control. Except for the 


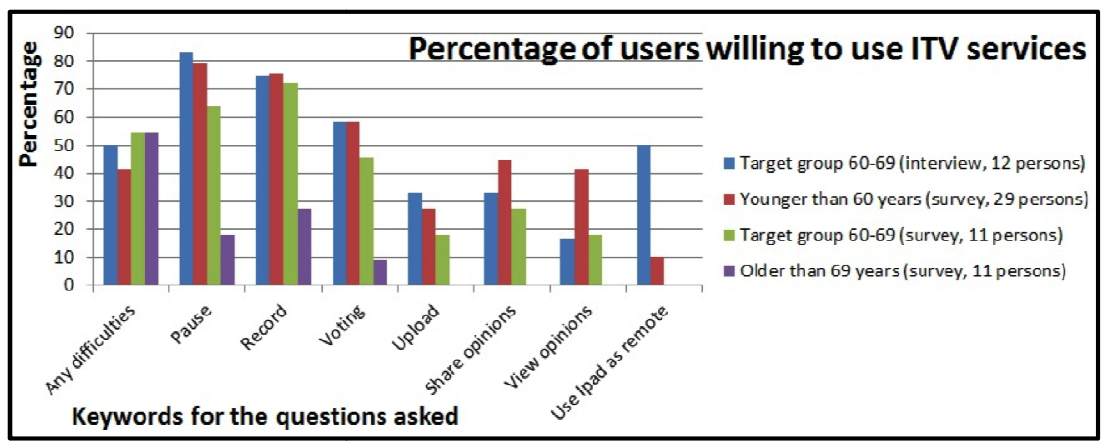

Fig. 3. Comparison of survey responses and interview for eight key questions

age group "Older than 69" the responses in general looks quite similar. Looking at the target age group (60-69), a close correspondence between the interview and survey can be observed, thus confirming the interview findings.

On the basis of Figure 3 it is interesting to see if any significant differences can be observed. An Exact Fischer test [Dalgaard, 1999] was carried out on the results of the questionnaire and revealed significant differences for six of the questions. A subsequent CHI-squared test showed that the group "older than 69" significantly stands out from the other three age groups. The age group "Younger than 60" wanted significantly more to e.g. "share opinions" and "view opinions" compared to the other age groups.

\subsection{User Requirements Derived from the Interviews}

A set of requirements derived from the analysis of the interviews can now be summarized and drawn up for the elderly (60-69 years):

- Easy and intuitive TV set-up configurations

- Better support

- Fewer remote controls

- The broadcaster should be accountable for any user contributed information

- No spam/interruptions

- No disturbing information on the TV screen

- Interacting in a familiar way

- In control of level of participation

- Information about new TV/iTV services

- Improved ease of use

- The user interface should adapt to a person's behavioural routine

\section{Design Recommendations for iTV Services for the Elderly}

The design recommendations derive from the requirements identified in Section 3. The requirements have been collapsed and translated into design recommendations 
according to well-established interaction design paradigms [Sharp et al 2011]. We propose the following ten design guidelines for the elderly:

1. Navigating the user interface should be without ambiguity and be highly transparent

2. A universal remote with a simple layout and easily detectable buttons should be used

3. Setting up the TV should be simplified and preferably employ a "conversing interface" design

4. The user interface should make use of red, orange and yellow colours with a high contrast between foreground and background.

5. Zooming of subtitles and enhancement of foreground sound should be supported

6. The user interface navigation should be easy, intuitive and follow a familiar pattern for TV interaction

7. It should be possible to hide/remove certain iTV services, such as social network integration

8. The interaction design should not allow for intrusive and disturbing content without the explicit permission from the user

9. Improved functions for channel sorting and personalisation

10. Adaptive interface: To accommodate the elderly, it will be beneficial to include several user interface profiles to choose among, at least one of them adhering to the above mentioned recommendations.

The final recommendation will enable designers to achieve the other nine without ending up with a need for producing TVs and iTV services especially targeted for the elderly.

\section{Conclusions}

The approach in this study is a qualitative research consisting of an explorative interview and a survey, which has proven suitable for exploring the elderly's opinions and experiences with TV and iTV services. The findings generated a number of user requirements, which, together with physiological and cognitive requirements, lead to a list of 10 design guidelines/ recommendations. Furthermore it was found that the age group from 60 to 69 years did not significantly stand out compared with the younger age groups, when asking about the willingness to use six common iTV services. It was found that those above 70 did stand out compared with the younger age groups - including the 60-69 years.

The outcome of this paper contributes to the design guidance for user interfaces for the elderly for iTV services.

Acknowledgements. The authors wish to thank Susan Möller Ferreira who is a Ph.D. student at the Pompeu Fabra University, Spain and a visiting colleague at Aalborg University for her collaboration with developing the interview guide and conducting two of the interviews. 


\section{References}

1. Cesar, P., Chorianopoulos, K.: The Evolution of TV Systems, Content, and Users Toward Interactivity. In: Foundations and Trends in Human-Computer Interaction, p. 97 (2008)

2. Dalgaard, P.: Introductory Statistics with R. Springer, New York (2008)

3. Fleury, A.: Near-Future Trends in Interactive Media Convergence Using Quantitative and Qualitative Approaches. PhD thesis, Aalborg University, Denmark (2012)

4. Gauntlett, D., Hill, A.: The retired and elderly audiences. In: TV Living: Television, Culture and Everyday Life, pp. 173-208 (1999)

5. Goldstein, E.B.: Sensation \& Perception. Vicki Knight (2007)

6. Kvale, S., Brinkmann, S.: InterView - Introduktion til et håndværk. Hans Reitzels Forlag, København (2009)

7. Livingstone, S.: The Challenge of Changing Audiences, Or What is the Audience Researcher to do in the Age of the Internet. European Journal of Communication 19(1), 75-86 (2004)

8. Madden, D.J.: Aging and Visual Attention. NIH Public Access - Author Manuscript 16(2), 70-74 (2007)

9. Morse, J.M., et al.: Verification Strategies for Establishing Reliability and Validity in Qualitative Research. International Journal of Qualitative Methods 1(2), 1-19 (2002)

10. Morse, J.M., Swanson, J.M., Kuzel, A.J.: The Nature of Qualitative Evidence. Sage Publications, Inc. (2001)

11. Obrist, M., Bernhaupt, R., Tscheligi, M.: Interactive TV for the Home: An Ethnographic Study on Users Requirements and Experiences. Intl. Journal of Human-Computer Interaction, 22 (2008)

12. Oppenheim, A.N.: Questionnaire Design, Interviewing and Attitude Measurement. Continuum, London (1992)

13. Schellenbach, M., et al.: Sensorimotor-Cognitive Couplings in the Context of Assistive Spatial Navigation for Older Adults. GeroPsych: The Journal of Gerontopsychology and Geriatric Psychiatry 23(2), 69-77 (2010)

14. Sharp, H., Yvonne, R., Preece, J.: Interaction Design - beyond human-computer interaction, 3rd edn. John Wiley \& Sons, England (2011)

15. Stirling, E.: Valuing Older People - Positive Psychological Practice. Wiley-Blackwell, West Sussex (2010)

16. Stuart-Hamilton, I.: Aldringens Psykologi. Gyldendal, Nordisk Forlag, København (2001)

17. Tsekleves, E., Whitham, R., Kondo, K., Hill, A.: Investigating media use and the television user experience in the home. Entertainment Computing, 10 (2011)

18. Weil, D.N. . Department of Economics, Brown University and NBER, Palgrave Encyclopedia of Economics, 2nd edn. (2006)

19. Whitbourne, S.K.: The Aging Individual - Physical and Psychological Perspectives. Springer Publishing Company, Inc., New York (2002)

20. Yin, R.K.: Qualitative Research from Start to Finish. The Guilford Press, New York (2011) 